\title{
Analyse der formalen Eigenschaften der Argumentation in deutschen und chinesischen wissenschaftlichen Texten
}

\author{
Hung-Cheng Liu (Chang-Hua)
}

\begin{abstract}
In scientific texts argumentation is a common tool. One has to prove ones position and perspective through arguments. The implementation of one's argumentation is the cultural and language specific conditions. In research literature, a comparison of the formal characteristics of scientific argumentation between the German and Chinese scientific texts is to my knowledge so far not yet available. Therefore, it is very interesting to compare the two languages with one another and to study the effect on language-specific particularities of an argumentation, as the formal properties of the argumentation are implemented differently.

The structure of an argumentation in German and Chinese is the focus of this work, since the argumentation in this regard isn't yet fully understood. In the present work some discourse markers indicating argumentative relations and consequences, are contrastively contrasted and compared with the frequency of their occurrence in texts. The following analysis has been based on five texts in German and Chinese. Its aim will be to how often the two conceptual categories - argumentative relation and argumentative consistency - occur in scientific texts. Through this comparison, some peculiarities of the formal properties of the argumentation will be presented.
\end{abstract}

\section{$1 \quad$ Einleitung}

Die Argumentation als Forschungsgegenstand ist in unterschiedlichen Disziplinen untersucht worden, wie z. B. in der Logik, der Politik und der Linguistik. Diese betrachten die Sprechhandlung Argumentation unterschiedlich und kommen deswegen $\mathrm{zu}$ sehr verschiedenen Ergebnissen (vgl. Wohlrapp 2008; Bayer 2007; Johnson 2006; Woods 2000; Willard 1996). Auf deren Ergebnisse soll in dieser Arbeit lediglich dahingehend verwiesen werden, dass sich der Autor ihrer bewusst ist, jedoch nicht auf diese rekurriert, da ihnen die kontrastive Perspektive, welche der vorliegenden Arbeit zugrunde liegt, fehlt.

In der Sprachwissenschaft gibt es zahlreiche Untersuchungen, welche die Argumentation unter einer kontrastiven Perspektive zum Gegenstand machen (vgl. Birial/Yakhabi 2013; Liu 2005; Hinkel 1999; Kim 1996; Bouchard 1996; Ferris 1994; Lux 1991; Choi 1988). Diese kontrastiven Forschungen haben rhetorische und inhaltliche Unterschiede und Gemeinsamkeiten aufgedeckt. Zum Beispiel finden Ferris (1994) und Hinkel (1999), dass englische Muttersprachler und ESL-Studenten sich bei der Verwendung von Gegenargumenten unterscheiden. Lux (1991) findet heraus, dass englischsprachige Studenten 
im Vergleich mit muttersprachlich Spanisch sprechenden Studenten vor allem in der schriftlichen Argumentation einen elaborierten Stil bevorzugen. Nach Liu (2005) einigen sich amerikanische und chinesische Gruppen auf den Zweck, eine dreigliedrige Struktur ${ }^{1}$ der Argumentationskette und die Verwendung formaler Logik, aber sie unterscheiden sich in der Diskussion über einige Grundlagen für argumentative Texte. Die chinesische Gruppe zeigt die Verwendung der epistemologischen und dialektischen Logik und unterstreicht die Notwendigkeit, Analogien zu verwenden. Dahingegen hält die amerikanische Gruppe die Vorwegnahme der Opposition für ein Muss. Obwohl die bisherigen Untersuchungen zahlreiche Ergebnisse über rhetorische und inhaltliche Unterschiede und Gemeinsamkeiten zwischen verschiedenen Sprachen aufgedeckt haben, liegt ein Vergleich zwischen deutschsprachigen und chinesischsprachigen Fachtexten in Bezug auf formale Eigenschaften der Argumentation in der Wissenschaftssprache noch nicht vor.

Das Ziel der vorliegenden Arbeit ist es, Unterschiede zwischen deutsch- und chinesischsprachigen Fachtexten hinsichtlich der formalen Eigenschaften der Argumentation herauszuarbeiten. Dabei soll aufgezeigt werden, wie die Argumentation jeweils formal unterschiedlich realisiert ist. Um diese Unterschiede herauszuarbeiten, werden Relationen der Argumentation auf der Satzebene und Konsequenzen der Argumentation auf der Textebene analysiert. Hierdurch soll ein Eindruck davon gewonnen werden, wie die formalen Eigenschaften der Argumentationen in der Wissenschaftssprache tatsächlich vorkommen. Da die vorliegende Arbeit als Voruntersuchung für eine weitere qualitative Analyse dient, wird auf Repräsentativität zunächst kein Schwerpunkt gelegt. Das Datenkorpus beschränkt sich daher im Deutschen und Chinesischen jeweils auf fünf Aufsätze. Die Ergebnisse wurden dann kontrastiv gegenübergestellt und verglichen.

\section{Theoretische Grundlagen}

Auf formaler Ebene bedeutet Argumentation die Verknüpfung mehrerer Argumente (vgl. Bayer 2007: 16). In wissenschaftlichen Texten gehört das Argumentieren zum Alltag. Jeder muss seine Position bzw. Perspektive mit Argumenten belegen. Wie man die Argumentation umsetzt, ist kultur- und sprachspezifisch bedingt. In diesem Kapitel werden einige Begriffe, die für die Analyse relevant sind, erläutert. Bei der Darstellung geht es auch darum, Analyseperspektive und Analysekategorien der vorliegenden Untersuchung herauszuarbeiten.

\subsection{Analyseperspektive}

In wissenschaftlichen Texten dient ein Argument vorwiegend zur Begründung von Behauptungen. Argument bedeutet hier Beweisgrund oder Beweismittel. Eine Argumentation zielt in der Wissenschaft hauptsächlich auf die Wahrheit der Ereignisse, in der Rhetorik aber auf die Überzeugung der Leser (vgl. Lumer 2007: 10). Durch diese Unterscheidung fanden rhetorische Analyseperspektiven in der vorliegenden Untersuchung keine Berücksichtigung.

\footnotetext{
1 Toulmin (1958: 88) spricht von einer dreigliedrige Struktur in der folgenden Argumentationskette: Harry hat die britische Staatsangehörigkeit (claim). Harry wurde auf den Bermudas geboren (data). Wer auf den Bermudas geboren wurde, bekommt die britische Staatsangehörigkeit (warrant).
} 
Der Aufbau der Argumentation, also die Perspektive der formalen Analyse, steht im Mittelpunkt dieser Arbeit, da die Argumentation in dieser Hinsicht kaum erforscht ist.

Die Forschungsgeschichte zum Gegenstand der Argumentation ist seit den Arbeiten von Toulmin (1958) und Perelman (1979) zu theoretischen Grundlagenüberlegungen dokumentiert. Seit Beginn der 90er Jahre wurde die Argumentation zunehmend zum Untersuchungsgegenstand empirisch arbeitender Sprachwissenschaftler. In der linguistischen Argumentationsforschung entwickelte sich das Verhältnis von Theorie und Empirie zu einem grundlegenden Problem, da sich abstrakte Argumentationstheorien insbesondere aus der Philosophie nur schwer und höchstens indirekt für die Analyse der linguistisch relevanten Aspekte von Argumentation nutzen lassen (Bücker 2004: 1). Als Beispiel dient Toulmins Modell (1958: 87-89) wie folgt:

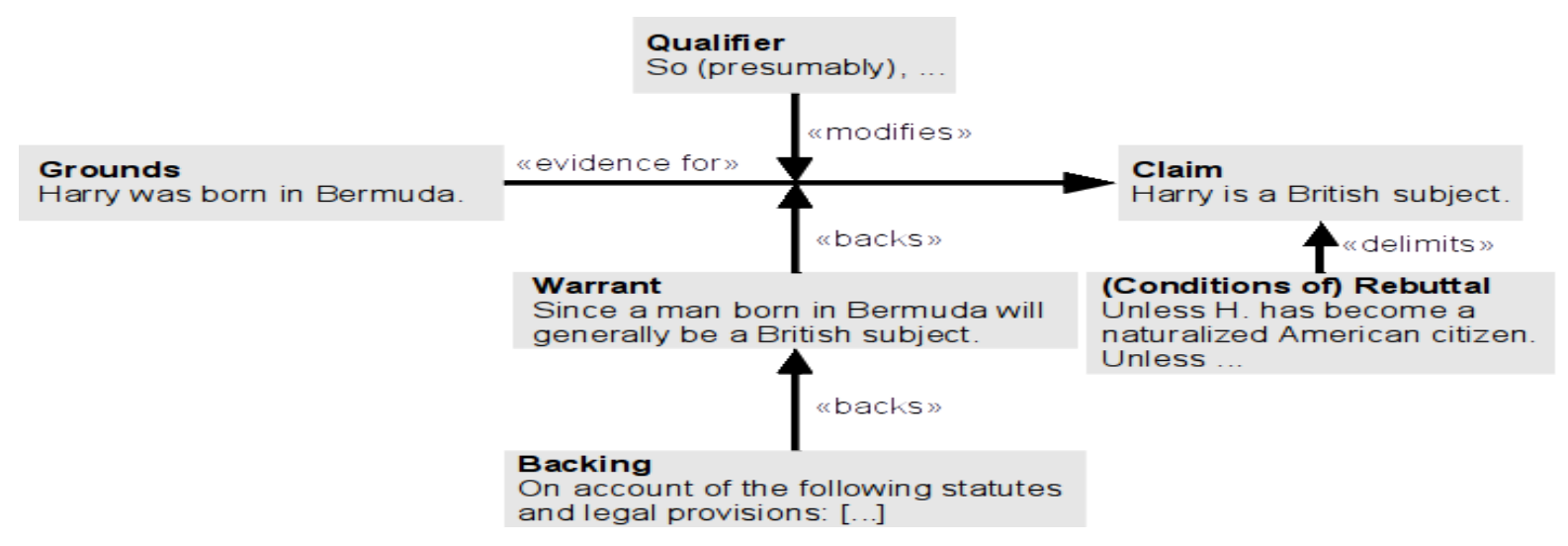

Abbildung 1: Toulmins Modell der Argumentation

Der britische Philosoph Stephen Toulmin bemerkt, dass gute realistische Argumente in der Regel aus sechs Teilen bestehen. Er verwendet diese Begriffe, um die Elemente zu beschreiben:

Data (Grounds): The facts or evidence used to prove the argument

Claim: The statement being argued (a thesis)

Warrants: The general, hypothetical (and often implicit) logical statements that serve as bridges between the claim and the data

Qualifiers: Statements that limit the strength of the argument or statements that propose the conditions under which the argument is true

Backing: Statements that serve to support the warrants (i.e., arguments that don't necessarily prove the main point being argued, but which do prove the warrants are true.)

Rebuttals: Counter-arguments or statements indicating circumstances when the general argument does not hold true.

(Toulmin 1958: 89)

Zahlreiche Forschungen basieren auf diesem Modell, allerdings lassen sich mit ihm die Forschungsergebnisse oft nur unzureichend beschreiben, da "Claim" normalerweise vor den Prämissen ("Data“) bzw. allein erscheint und eine Einschränkung („Qualifiers”) selten vorkommt. Das Modell von Toulmin ist schließlich ungenau hinsichtlich der Frage, welcher Typ von argumentativer Handlung in diesem Modell repräsentiert werden soll (Kindt 2008: 151). Weiterhin versucht Walton (2008, 2012), anstatt des Toulmin-Modells die Schemata der Argumentation $\mathrm{zu}$ analysieren. Er hat insgesamt achtundneunzig Muster zusammengestellt. Der Nachteil dieser Sammlung von Argumentationsmustern liegt darin, 
dass sie zu trivial und aufwendig sind, als dass sie als universelles Argumentationsmuster Anwendung finden könnten. Vereinfacht hat die automatische Argumentationsanalyse der Arbeit von Kluge gezeigt, dass man die zehn häufigsten Argumentationsmuster kontroverser Bildungsthemen entsprechend ihrem Vorkommen in Webdokumenten wie folgt formulieren kann (Kluge 2014: 44).

$\begin{array}{llll}\text { Rangfolge } & \text { Formel } & \text { Häufigkeit } & \text { Prozentualer Anteil } \\ \text { 1. } & C \rightarrow S-P o & 56 & 31.3 \% \\ \text { 2. } & S-P r \rightarrow C & 42 & 23.5 \% \\ \text { 3. } & C & 39 & 21.8 \% \\ \text { 4. } & A-P r \rightarrow C & 9 & 5.0 \% \\ \text { 5. } & C \rightarrow S-P O \rightarrow A-P o & 6 & 3.4 \% \\ \text { 6. } & A-P r \rightarrow C \rightarrow S-P o & 5 & 2.8 \% \\ \text { 7. } & S \rightarrow P r \rightarrow C \rightarrow S-P o & 5 & 2.8 \% \\ \text { 8. } & C \rightarrow A-P O \rightarrow S-P O & 4 & 2.2 \% \\ \text { 9. } & A-P r \rightarrow S-P r \rightarrow C & 3 & 1.7 \% \\ \text { 10. } & S-P r \rightarrow C \rightarrow A-P o & 3 & 1.7 \% \\ \text { (Zeichenerklärung: } \mathrm{C}-\text { claim, C-Re }- \text { restatement, A/S-Pr/Po- attack/support- pre/post-claim) }\end{array}$

Wenn man die automatische Erkennung genau ansieht, bekommt man den Eindruck, dass man durch eine solche Analyse nur die Reihenfolge von einzelnen Elementen der Argumentation festlegt. Durch eine solche Vorgehensweise kann man keinen Einblick in die sprachliche Verwendung der formalen Eigenschaften der Argumentation erhalten.

Wie bereits am Anfang dieses Kapitels erwähnt, bezeichnet Argumentation auf der formalen Ebene die Verknüpfung mehrerer Argumente (vgl. Bayer 2007: 16). Diese formale Bedeutung der Argumentation kann auf der Satzebene als argumentative Relation und auf der Textebene als argumentative Konsequenz bezeichnet werden. Im nächsten Abschnitt wird darauf eingegangen, welche formalen Eigenschafen der Argumentation in Bezug auf argumentative Relationen und Konsequenzen zum Ausdruck kommen können. Hier muss darauf hingewiesen werden, dass die folgenden zwei Analysekategorien: argumentative Relation und argumentative Konsequenz einschließlich ihrer jeweiligen Subkategorien vom Autor als Konzept für die vorliegende Untersuchung entwickelt wurden.

\subsection{Analysekategorien}

Argumentative Relation: Die argumentative Relation, die wir hier als Analysekategorien verwenden, bezieht sich auf die Verknüpfung zwischen Claim und Prämisse auf der Satzebene. Dabei kann man diese Relation unter verschiedenen Konstellationen erschließen. $\mathrm{Zu}$ nennen sind: begründende, inferentielle, bedingungsbezeichnende sowie einschränkende 
Relationen. Diese Relationen können durch Diskursmarker ${ }^{2}$ erschlossen werden. Gruber, Rheindorf und Wetschanow et al. (2006: 183) haben eine Reihe von Wörtern klassifiziert, welche verschiedene Relationen der Argumentation signalisieren. Nach deren Klassifikation werden mit der folgenden Tabelle Diskursmarker dahingehend modifiziert dargestellt, dass sie argumentative Relationen signalisieren.

\begin{tabular}{|c|c|c|}
\hline Relationen auf der Satzebene & \multicolumn{2}{|c|}{ Signalisierende Mittel } \\
\hline \multirow[t]{5}{*}{ Begründende Relation } & weil & 因為 \\
\hline & denn & 由於 \\
\hline & deswegen & 正因如此 \\
\hline & nämlich & 就是 \\
\hline & aus diesem Grund & 基於這個原因 \\
\hline \multirow[t]{4}{*}{ Inferentielle Relation } & so dass & 所以 \\
\hline & folglich & 因而 \\
\hline & infolgedessen & 之所以 \\
\hline & deshalb & 因此 \\
\hline \multirow[t]{4}{*}{ Bedingungsbezeichnete Relation } & wenn & 當 \\
\hline & falls & 如果 \\
\hline & andernfalls & 不然 \\
\hline & sonst & 否則 \\
\hline \multirow[t]{5}{*}{ Einschränkende Relation } & obwohl & 雖然 \\
\hline & allerdings & 不過 \\
\hline & trotzdem & 儘管如此 \\
\hline & jedoch & 然而 \\
\hline & lediglich & 只/只是 \\
\hline
\end{tabular}

Tabelle 1: Signalisierende Mittel für Relationen auf der Satzebene

Die oben dargestellten deutschen Diskursmarker beanspruchen keine Vollständigkeit in der jeweiligen Analysekategorie. Auf sie sollen Texte probeweise dahingehend durchsucht werden, ob die unterschiedlichen Analysekategorien unabhängig von ihrem jeweiligen Inhalt in wissenschaftlichen Texten tatsächlich vorkommen. Um eine Vergleichsbasis zu schaffen,

\footnotetext{
${ }^{2}$ Swan (2005: 18) definiert einen „Diskursmarker“ als ein Wort oder einen Ausdruck, der die Verbindung zwischen dem, was gesagt wird, und dem größeren Zusammenhang zeigt. Demnach ist ein Diskursmarker etwas, das entweder ein Satz mit Vorangegangenem oder Nachkommendem verbindet, oder aber die Haltung des Autors zum Gesagten aufzeigt.
} 
sind die oben präsentierten chinesischen Diskursmarker in Übersetzung und unter Berücksichtigung ihrer Funktions- und Bedeutungsäquivalenz parallel gelistet. Die Stelle, an der die Diskursmarker gefunden werden, wird mit einer Markierung für die jeweilige argumentative Relation gekennzeichnet. Auf diese Weise kann die Anzahl der einzelnen argumentativen Relationen in beiden Sprachen empirisch erfasst werden.

\section{- $\quad$ Argumentative Konsequenz}

Des Weiteren können argumentative Konsequenzen auf der Textebene je nach ihrer Struktur in Subkategorien gefasst werden. $\mathrm{Zu}$ nennen sind lineare und erhaltende Strukturen. Die folgende Abbildung stellt dar, wie verschiedene Argumente strukturiert werden können (Liu 2000: 79).

\section{$\bullet \rightarrow O^{\uparrow} \cdot \rightarrow \bullet \rightarrow O_{\downarrow} \rightarrow \bullet \bullet \rightarrow$}

Abbildung $2^{3}$ : Argumentative Konsequenzen auf der Textebene

Eine lineare Struktur bezeichnet eine Kettenstruktur, in der Argumente nach einander folgen. Diese Struktur wird als anreihende oder ergänzende Konsequenz bezeichnet. Die beiden Konsequenzen folgen dem Prinzip der horizontalen Progression. Die Frage, ob sie untereinander austauschbar bzw. durcheinander ersetzbar sind, lässt sich anhand der folgenden Beispiele aufzeigen.

\section{- $\quad$ Anreihende Konsequenz}

Der zentrale Kritikpunkt der Online-Befragung berührt in erster Linie die inferenzstatistische Auswertung der Daten (vgl. Tabelle 2). Damit von einer Stichprobe auf die Grundgesamtheit geschlossen werden kann, muss erstens die Grundgesamtheit definiert sein und zweitens eine repräsentative, d. h. entweder zufällig ausgewählte oder systematisch gezogene Stichprobe vorliegen.

(Juska-Bacher 2013: 11)

\section{- $\quad$ Ergänzende Konsequenz}

Als Funktionsverben gelten hier nach Heine (2004: 74): bringen, finden, gehen, halten, kommen, nehmen, setzen, stehen, stellen, treten und ziehen. Aus technischen Gründen musste außerdem das Verb machen von der Analyse ausgeschlossen werden, da hierfür keine Daten in der Kollokationsdatenbank des DWDS vorliegen.

(Möhring 2011: 35)

Eine Unterscheidung kann dahingehende unternommen werden, dass die ,anreihende Konsequenz" eine punktuelle Verortung, die „ergänzende Konsequenz“ hingegen, eine aspektuelle Verortung auf der Textebene bedingt. Beide ähneln sich zwar in ihrer horizontalen Progression, unterscheiden sich aber in ihrer jeweiligen sprachlichen Orientierung.

Ferner kommt eine erhaltende Struktur vor, wenn die Kettenstruktur unterbrochen ist. Dabei werden zusätzliche Informationen geliefert, um etwas zu verstärken bzw. zu verweisen. Diese Struktur wird in der vorliegenden Arbeit als verstärkende oder verweisende Konsequenz

\footnotetext{
${ }^{3}$ Zeichenerklärung: Die nacheinander folgenden Pfeile , $\rightarrow \rightarrow \rightarrow$ “ werden hier als lineare Struktur bezeichnet, wobei das Zeichen „• $\rightarrow$ “ auf eine punktuelle Perspektive und das Zeichen „ $\rightarrow$ " auf eine aspektuelle Perspektive hinweist. Ferner bedeutet der Kreis „O“ eine erhaltende Struktur, indem das Zeichen „O^“ eine hervorhebende Perspektive und das Zeichen „O\“ für eine vertiefende Perspektive besitzt.
}

ISSN 1615-3014 
bezeichnet. Die beiden Konsequenzen folgen dem Prinzip der vertikalen Progression. Im Gegensatz zur horizontalen Progression bedeutet die vertikale Progression, dass die Perspektive der horizontalen Progression abgelöst und durch einen anderen Blickwinkel ersetzt wird. Dies kann anhand der folgenden Beispiele veranschaulicht werden:

\section{- Verstärkende Konsequenz}

Die Systematik schließt auch verschiedene für gesprochene Sprache typische Formen wie Topikalisierungen und elliptische Frageformulierungen ein und wird im Folgenden noch einmal exemplifiziert:

(Rost-Roth 2011: 103)

\section{- Verweisende Konsequenz}

Daraus entstehen dann Resultats- und Vergangenheitslesarten. In (1) und (3) macht der Sprecher eine Aussage über den Nachzustand, der in der Gegenwart (jetzt) bzw. in der Zukunft (gleich) liegt. Die resultative Gegenwartslesart lässt sich beispielsweise wie folgt veranschaulichen:

(Schumacher 2011: 66)

Der Unterschied zwischen den beiden besteht darin, dass die erste Ausdrucksweise eine verstärkende Perspektive und die zweite eine verweisende Perspektive einnimmt, daraus ergeben sich eine verstärkende und eine verweisende Konsequenz.

Mit der folgenden Tabelle wird verdeutlicht, welche Diskursmarker argumentative Konsequenzen signalisieren.

\begin{tabular}{|l|l|l|}
\hline Konsequenzen auf der Textebene & \multicolumn{2}{|c|}{ Signalisierende Mittel } \\
\hline Lineare Struktur: \\
Die anreihende Konsequenz & $\begin{array}{l}\text { erstens, zweitens, } \\
\text { drittens }\end{array}$ & 第一, 第二, 第三 \\
\cline { 2 - 3 } & zuerst & 首先 \\
\cline { 2 - 3 } & abschließend & 總之 \\
\cline { 2 - 3 } & schließlich & 最後 \\
\hline Lineare Struktur: & des Weiteren/ weiter & 進一步 \\
\cline { 2 - 3 } Die ergänzende Konsequenz & außerdem & 此外 \\
\cline { 2 - 3 } & ferner & 另外 \\
\cline { 2 - 3 } & zusätzlich & 附加 \\
\cline { 2 - 3 } & darüber hinaus & 另一方面 \\
\hline \multirow{5}{*}{$\begin{array}{l}\text { Erhaltende Struktur: } \\
\text { Die verstärkende Konsequenz }\end{array}$} & besonders & 特別的是 \\
\cline { 2 - 3 } & zu betonen & 強調 \\
\cline { 2 - 3 } & noch einmal & 再者 \\
\cline { 2 - 3 } & vor allem & 特別是 \\
\cline { 2 - 3 } & insbesondere & 尤其是 \\
\hline
\end{tabular}




\begin{tabular}{|c|c|c|}
\hline \multirow{5}{*}{$\begin{array}{l}\text { Erhaltende Struktur: } \\
\text { Die verweisende Konsequenz }\end{array}$} & zum Beispiel/ wie z. B. & 例如 \\
\hline & das heißt & 就是說 \\
\hline & auf diese Weise & 依照 \\
\hline & beispielsweise & 比如 \\
\hline & so wie & 如 \\
\hline
\end{tabular}

Tabelle 2: Signalisierende Mittel für Konsequenzen auf der Textebene

Ähnlich wie argumentative Relationen beanspruchen die oben dargestellten deutschen Diskursmarker keine Vollständigkeit in der jeweiligen Subkategorie. Auf sie sollen Texte probeweise durchsucht werden. Um eine Vergleichsbasis $\mathrm{zu}$ schaffen, sind die oben präsentierten chinesischen Diskursmarker in Übersetzung und unter Berücksichtigung ihrer Funktions- und Bedeutungsäquivalenz parallel gelistet. Die Stelle, an der die Diskursmarker gefunden werden, wird mit einer Markierung für die jeweilige argumentative Konsequenz gekennzeichnet. Auf diese Weise kann die Anzahl der einzelnen argumentativen Konsequenzen in beiden Sprachen empirisch erfasst werden.

\section{Methodische Vorgehensweise}

Hier muss darauf hingewiesen werden, dass die vorliegende Arbeit sich als quantitative Untersuchung versteht und als Voruntersuchung für eine weitere qualitative Analyse dienen soll. Von daher wird kein Kontextbezug in die Interpretation miteinbezogen und kein Schwerpunkt auf Repräsentationsfähigkeit gelegt.

\subsection{Korpus}

Das deutsche Korpus wurde Linguistik Online entnommen. Von den in der Quelle vorhandenen insgesamt dreißig Texten wurden lediglich die fünf von Muttersprachlern geschriebenen Texte ausgewählt. Parallel gab es dreißig chinesische Texte, die von der linguistischen Zeitschrift Science Sinica Taiwans ausgewählt wurden. Um eine Vergleichsbasis zu schaffen, wurden von den chinesisch-sprachigen Texten ebenfalls nur fünf zur Analyse gestellt. Die Texte wurden wie folgt zusammengestellt:

\begin{tabular}{|l|l|}
\hline \multicolumn{1}{|c|}{ Deutsche Texte } & \multicolumn{1}{c|}{ Chinesische Texte } \\
\hline $\begin{array}{l}\text { Möhring, Jupp: „Kollokationen } \\
\text { im Lernerwörterbuch - Anspruch } \\
\text { und Wirklichkeit“. Linguistik } \\
\text { online 47: 3/11. }\end{array}$ & $\begin{array}{l}\text { 鄭秋豫 (2010): 語篇的基頻構組與語流韻律體現 } \\
\text { (“An Analysis of Discourse Construction and Global } \\
\text { Information in Realized Narrative Prosody”). } \\
\text { Language and linguistics 11/2: 183-218. }\end{array}$ \\
\hline $\begin{array}{l}\text { Rost-Roth, Martina: „Formen und } \\
\text { Funktionen von Interrogationen“. } \\
\text { Linguistik online 49: 5/11. }\end{array}$ & $\begin{array}{l}\text { 魏培泉(2010): 「是否-V(N)P 」句式的由來 (“The } \\
\text { History of the Interrogative Structure shi fou-V(N)P”). } \\
\text { Language and linguistics 11/2: 335-392. }\end{array}$ \\
\hline $\begin{array}{l}\text { Schumacher, Nicole: } \\
\text { „Nachzustand, Distanz und }\end{array}$ & 張麗麗(2010): 返回義趨向詞作狀語--從語義框架看 \\
\hline
\end{tabular}




\begin{tabular}{|c|c|}
\hline $\begin{array}{l}\text { Aspektualität“. Linguistik online } \\
\text { 49:5/11. }\end{array}$ & $\begin{array}{l}\text { 虛化 (“Directionals Expressing the Notion of "Return" } \\
\text { as Adjuncts - A Study of Grammaticalization from the } \\
\text { Perspective of Semantic Frame"). Language and } \\
\text { linguistics 11/4: 803-851. }\end{array}$ \\
\hline $\begin{array}{l}\text { Hartung, Thomas: „Die } \\
\text { Paronomasie als } \\
\text { werbestilistisches Element““. } \\
\text { Linguistik online } 55,5 / 12 \text {. }\end{array}$ & $\begin{array}{l}\text { 蕭惠貞/林倩如 (2012): 論現代漢語補語可能構式 } \\
\text { (“On Mandarin Potential Complement Constructions”). } \\
\text { Language and linguistics 13/5: 963-998. }\end{array}$ \\
\hline $\begin{array}{l}\text { Juska-Bacher, Britta: } \\
\text { „Webbasierte linguistische } \\
\text { Forschung“. Linguistik online 61: } \\
4 / 13 \text {. }\end{array}$ & $\begin{array}{l}\text { 洪惟仁 (2013): 台灣的語種分布與分區 (“The } \\
\text { Distribution and Regionalization of Varieties in } \\
\text { Taiwan”). Language and linguistics 14/2: 315-369. }\end{array}$ \\
\hline
\end{tabular}

Tabelle 3: Quellenangaben für Textkorpus im Deutschen und Chinesischen

\subsection{Quantitative Datenanalyse}

Die quantitative Datenanalyse führt dazu, dass man zuerst einen Überblick über die gesamte Analyse erhält. Das hier verwendete Tool heißt AntConc ${ }^{4}$, welches es erlaubt eine Wortgruppe in den Texten zu suchen. Ein Beispiel aus der Analysekategorie „begründende Relation“ soll das Arbeitsfenster und den Suchvorgang sowie das Suchergebnis in AntConc veranschaulichen. Zuerst wurden die Diskursmarker - weil, denn, deswegen, nämlich und aus diesem Grund - eingegeben (Abbildung 3) und dann wurden die Texte auf sie hin durchsucht. Dies ergab folgendes Suchergebnis (Abbildung 4).

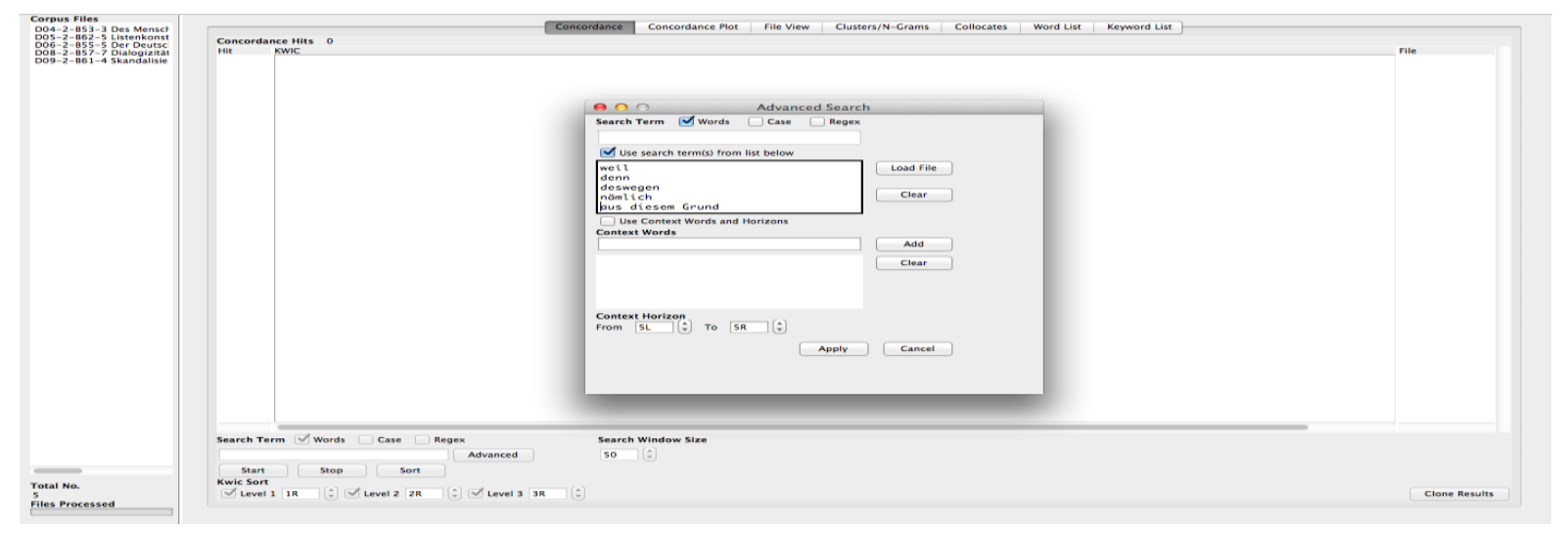

Abbildung 3: Angaben von Wortgruppe

\footnotetext{
${ }^{4}$ Das Tool wurde von Laurence Anthony entwickelt (vgl. www.laurenceanthony.net/contact.html).
} 


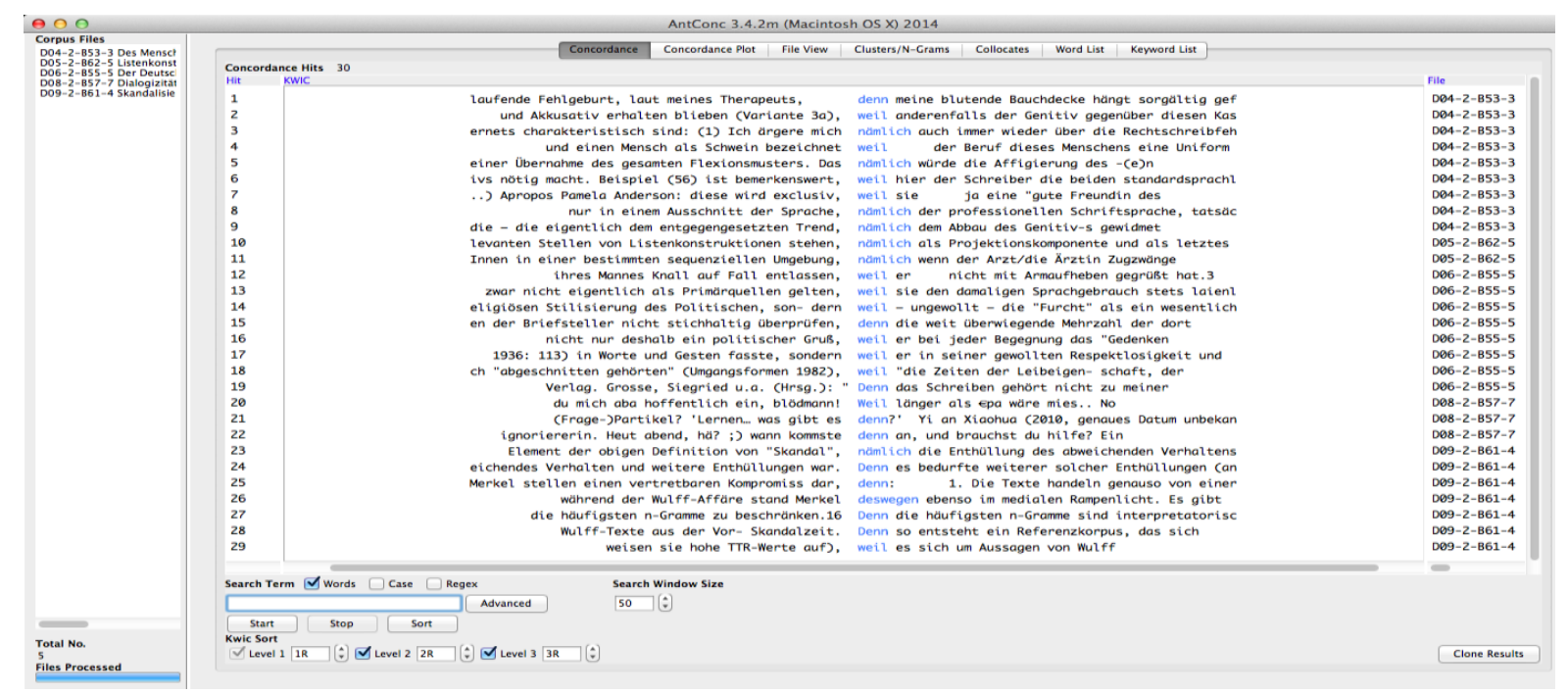

Abbildung 4: Das Suchergebnis

Die in der Tabelle 1 und 2 in Übersetzung dargestellten chinesischen Wörter wurden ebenfalls zuerst durch dieses Tool getestet. Dabei wurde zunächst einmal festgestellt, dass sie in den zu untersuchenden Texten vorkamen. Damit war die Funktionsäquivalenz mit der Übersetzung als Vergleichsbasis gesichert. So kann z. B. die deutsche Ausdrucksform der bedingungsbezeichneten Relation falls ins Chinesische mit 假如 (,Giaru') oder 如果 (,Ruguo') übersetzt werden. Basierend auf dem Test wurde lediglich der Diskursmarker 如果 (,Ruguo') ausgewählt, da er in den zu untersuchenden Texten auch tatsächlich vorkam.

\section{$4 \quad$ Ergebnisse}

\subsection{Argumentative Relationen auf der Satzebene}

Durch die im letzten Kapitel dargestellte Vorgehensweise sind die Suchergebnisse in den beiden Sprachen nun wie folgt zusammengestellt:

\begin{tabular}{|c|c|c|c|c|}
\hline \multirow[t]{2}{*}{ Relationen auf der Satzebene } & \multicolumn{2}{|c|}{ Deutsche Texte } & \multicolumn{2}{|c|}{ Chinesische Texte } \\
\hline & & & & \\
\hline & & & & \\
\hline \multicolumn{5}{|c|}{$\begin{array}{l}\text { Resultatslesarten weisen oft telische Ausdrücke auf, weil sich in der Verbsemantik } \\
\text { verankerte inhärente Endpunkte und ihre Nachzustände besonders gut dafür eignen, ins } \\
\text { Zentrum der Aussage gerückt zu werden. } \\
\text { 此一限制也正好能夠說明為何「反」和 }\ulcorner\text { 回」沒有發展出此一用法，因為「反 } \\
\lrcorner \text { 和 }\ulcorner\text { 回」的及物用法較不發達，不具備此項虛化條件。 } \\
\text { [Diese Unterteilung geschieht auch gerade deshalb, um zu klären, warum die } \\
\text { Ausdrücke Fan (,gegen') und Hue (,zurück') keine solche Verwendung finden. Weil } \\
\text { der transitive Gebrauch von Fan (,gegen`) und Hue (,zurück') weniger ausgeprägt ist, } \\
\text { hat er auch nicht dieselbe imaginäre Konnotation.] }\end{array}$} \\
\hline
\end{tabular}


Chinesische Texte: Aus der Tabelle lässt sich ablesen, dass die formale Eigenschaft der Argumentation im Chinesischen besonders häufig der inferentiellen Relation zu dienen scheint. Bemerkenswert ist hierbei, dass im Deutschen hingegen der Kategorie inferentielle Relation die niedrigste Häufigkeit zukommt.

Vergleich: Aus der Tabelle lassen sich zwei Ergebnisse ableiten. Das erste Ergebnis ist, dass im Deutschen mehr Wert auf konditionelle Relationen gelegt wird, während das Chinesische $\mathrm{zu}$ inferentiellen Relationen neigt. Das zweite Ergebnis ist, dass im Deutschen mehr bedingungsbezeichnende und einschränkende Relationen zum Ausdruck gebracht werden. Dagegen spielen diese Relationen im Chinesischen keine bedeutende Rolle.

Aus diesen beiden Ergebnissen lässt sich annehmen, dass die formale Eigenschaft der Argumentation im Chinesischen eine vorwärtsblickende Perspektive (inferentiell) und im Deutschen eine zurückblickende Perspektive (konditionell) bevorzugt.

\subsection{Argumentative Konsequenzen auf der Textebene}

Entsprechend dem letzten Abschnitt, sind die Untersuchungsergebnisse der beiden Sprachen nun wie folgt zusammengestellt:

\begin{tabular}{|l|c|c|c|c|}
\hline \multirow{2}{*}{ Konsequenz auf der Textebene } & \multicolumn{2}{|c|}{ Deutsch } & \multicolumn{2}{c|}{ Chinesisch } \\
\cline { 2 - 5 } & Anzahl & Häufigkeit & Anzahl & Häufigkeit \\
\hline anreihende Konsequenz & 9 & $0.35 \%$ & 21 & $0.76 \%$ \\
\hline
\end{tabular}

In (9) schließlich haben wir ein typisches Beispiel für die Verwendung des Präteritums in literarischen Texten. Es handelt sich um den Beginn von Alessandro Bariccos Questa Storia in seiner deutschen Übersetzung.

方式副詞「反」可表達兩種概念。首先, 「反」可以表示反向執行該動作，

例如先秦一些「反走」乃基於禮節表現出退避的行止, 是退走的意思。

[Das Modaladverb Fan (,gegen') kann zwei Konzepte auszudrücken. Erstens kann mit Fan (,gegen') die Umkehrung einer Aktion ausgedrückt werden, so sind beispielsweise in der frühen Qin Dynastie einige „Rückzüge“ der damaligen Etikette geschuldet.]

\begin{tabular}{|l|l|l|l|l}
\hline ergänzende Konsequenz & 24 & $0.93 \%$ & 30 & $1.09 \%$ \\
\hline
\end{tabular}

Es dürfte deutlich geworden sein, dass primäre Paronomasien nicht nur multilingual und multidimensional alle bis dato erarbeiteten un- oder spezifischen werbestilistischen Funktionen bedienen, sondern darüber hinaus linguistische Horizonte eröffnen, die auch und vor allem hinter den Landschaften der „klassischen“ Werbung Sehnsuchtsorte aufschließen.

$\mathrm{V}(\mathrm{N}) \mathrm{P}\lrcorner$ 的南方方言至少包括閩語、客語、粵語、吳語、湘語等。此外, 與客語 關係密切的粵北土話、番語也使用這種句式。

[Unter den südlichen Dialekten wie Min, Hakka, Kantonesisch, Wu und Xiang ist das Satzmuster „V (N) P“ gebräuchlich. Darüber hinaus verwendet der mit dem Hakka eng verwandte nördliche Guangdong-Dialekt ebenfalls ein solches Satzmuster.] 


\begin{tabular}{|c|c|c|c|c|}
\hline & 57 & & 5 & \\
\hline \multicolumn{5}{|c|}{$\begin{array}{l}\text { Unterschiede zeigen sich jedoch in den Frequenzen. Auffallend sind vor allem höhere } \\
\text { Anteile von elliptischen Strukturen und V2-Strukturen bei Nachfragen. } \\
\text { 只是作者難以同意這個看法。一者, 近代漢語助詞「也」的用法難以和上古漢語相提 } \\
\text { 並論；再者，此例按文義是向皇帝請示應否禁止所指錢幣的通行，也不應該是表達否 } \\
\text { 定的陳述句。 } \\
\text { [Der Verfasser kann nur schwer mit dieser Sichtweise einverstanden sein. Da die } \\
\text { Verwendung des Partikel Ye (,auch`) im modernen Chinesisch nicht mit der Verwendung im } \\
\text { klassischen Chinesisch gleich zu setzen ist; insbesondere ist der Kontext entscheidend für die } \\
\text { Interpretation, ob der Kaiser den Gebrauch von Münzen verbieten lassen sollte. Es handelt } \\
\text { sich dabei nicht um eine negative Aussage.] }\end{array}$} \\
\hline & & & & \\
\hline \multicolumn{5}{|c|}{$\begin{array}{l}\text { Um die Bedeutungs- und Gebrauchskomponenten Nachzustand, Distanz und Aspektualität i } \\
\text { einer formfokussierten Steuerung von Perfekt und Präteritum zu vermitteln, bieten sich - } \\
\text { was die Sprachrezeption betrifft - beispielsweise die Verfahren der Inputflut und des input } \\
\text { enhancement an. } \\
\text { 我們採人工方式判別詩歌的工整性, 依據準則為：(1) 標點 } \\
\text { 符號。採用較大的段落標點符號, 如：句號, 將文本分成包含多短語的段落區塊； } \\
\text { (2) 短語長度。 } \\
\text { [Ob die Poesie ordentlich und sauber ist, wird nach den folgenden Richtlinien entschieden: } \\
\text { (1) Die Interpunktion. Die Zeichensetzung wird für größere Passagen eingesetzt, wie zum } \\
\text { Beispiel: ein Punkt, der den Text in absatzweise mehrere Sätze unterteilt; } \\
\text { (2) die Länge des Satzes.] }\end{array}$} \\
\hline
\end{tabular}

\section{Tabelle 5: Argumentative Konsequenzen im Deutschen und Chinesischen}

Deutsche Texte: Erstaunlicherweise kommen beide Sprachen in der Kategorie ergänzende Konsequenz auf eine nahezu identische Anzahl an Diskursmarkern. Allerdings kommt die anreihende Konsequenz im Deutschen wenig vor. Daher kann man annehmen, dass die formale Eigenschaft der Argumentation im Deutschen auf der Textebene mehr der aspektuellen Verortung (ergänzende Konsequenz) dient.

Chinesischen Texte: Im Vergleich mit deutschen Texten neigen chinesische Texte mehr zu einer punktuellen Verortung (anreihende Konsequenz). Eine überdurchschnittliche Anzahl an Diskursmarkern lässt sich der Kategorie verweisende Konsequenz zuordnen. Dieser gravierende Unterschied bezeichnet die Eigenschaft der Argumentation im Chinesischen als vertikale Progression mit einer verweisenden Perspektive auf der Textebene.

Vergleich: Genau wie die Relationen auf der Satzebene, sind alle argumentativen Konsequenzen auf der Textebene für beide Sprachen relevant und vorhanden. Allerdings finden Sie unterschiedliche Gewichtung. Es lassen sich zwei Ergebnisse feststellen. Erstens 
orientiert sich das Chinesische mehr an der anreihenden Konsequenz, die eine Eigenschaft der punktuellen Orientierung besitzt, während dies im Deutschen nicht der Fall ist. Zweitens findet im Deutschen die verstärkende Konsequenz mehr Anwendung, während im Chinesischen die verweisende Konsequenz am häufigsten verwendet wird.

\section{$5 \quad$ Fazit}

Wie bereits in der Einleitung erwähnt, liegt trotz zahlreicher kontrastiver Untersuchungen ein Vergleich der formalen Eigenschaften der Argumentation im Deutschen im Vergleich zum Chinesischen Sprachgebrauch bisher nicht vor. Das Ziel der vorliegenden Arbeit besteht darin, anhand der konzeptuellen Kategorien - argumentative Relation und argumentative Konsequenz - die formalen Eigenschaften der Argumentation in den beiden Sprachen quantitativ zu erforschen. Die Ergebnisse werden nun im Folgenden zusammengestellt.

1. Im Deutschen wird mehr Wert auf konditionelle Relationen gelegt, während im Chinesischen inferentielle Relationen häufiger Ausdruck finden.

2. Im Deutschen finden in der Argumentation eher bedingungsbezeichnende und einschränkende Relationen Anwendung. Dagegen spielen diese Relationen im Chinesischen keine bedeutende Rolle.

3. Das Chinesische orientiert sich mehr an der anreihenden Konsequenz, während dies im Deutschen nicht der Fall ist.

4. Im Chinesischen findet die verweisende Konsequenz mehr Anwendung als im Deutschen. Dahingegen folgt das Deutsche der verstärkenden Konsequenz mehr als das Chinesische.

Durch die oben dargestellten Ergebnisse lassen sich einige Annahmen über die formalen Eigenschaften der Argumentation in den beiden Sprachen ableiten. Erstens, das Chinesische bevorzugt auf der Satzebene eine formale Eigenschaft mit einer vorwärtsblickenden Perspektive (inferentiell); das Deutsche hingegen eine zurückblickenden Perspektive (konditionell). Zweitens orientiert sich das Deutsche auf der Textebene mehr an einer aspektuellen Perspektive (ergänzende Konsequenz), das Chinesische hingegen eher an einer punktuellen Perspektive (anreihende Konsequenz). Drittens findet sich im Chinesischen auf der Textebene häufiger eine verweisende Perspektive (verweisende Konsequenz) und im Deutschen eher eine verstärkende Perspektive (verstärkende Konsequenz).

Die oben genannten Annahmen sollen in der Zukunft mit einer qualitativen Datenanalyse weiter überprüft werden. Es soll festgestellt werden, ob alle gekennzeichneten Stellen für die jeweiligen Kategorien zutreffend sind. Außerdem sollen mehr Texte in die Analyse miteinbezogen werden, um eine quantitativ legitimierte Analyse zu ermöglichen. Ob die Ergebnisse auch in der Forschung von Genreaspekten bzw. Fremdsprachendidaktik Anwendung finden können, wird Untersuchungsgegenstand zukünftiger Forschung sein.

\section{Literaturverzeichnis}

Baleghizadeh, Sasan/Pashaii, Azar (2010): "A comparison of English and Farsi rhetoric and its impact on English writing of Iranian students". Studies in Literature and Language 1/5: $17-27$. 
Bayer, Klaus (2007): Argument und Argumentation. Logische Grundlagen der Argumentationsanalyse. Göttingen: Vandenhoeck/Ruprecht.

Biria1, Reza/Yakhabi, Masoume (2013): “Contrastive Rhetorical Analysis of Argumentation Techniques in the Argumentative Essays of English and Persian Writers". Journal of Language, Culture, and Translation 2/1: 1-14.

Bücker, Jörg (2004): Argumentationstheorie und interaktionale Linguistik. Münster. (= SASI Heft 1). http://noam.uni-muenster.de/sasi/Buecker_SASI.pdf [15.2.2015].

Choi, Yeon Hee (1988): “Text structure of Korean speakers' argumentative essays in English". World Englishes 7/2: 129-137.

Ferris, Dana R. (1994): "Rhetorical strategies in student persuasive writing: Differences between native and non-native English speakers". Research in the Teaching of English 28: 45-61.

Gruber, Helmut/Rheindorf, Markus/Wetschanow, Karin et al. (2006): Genre, Habitus und wissenschaftiches Schreiben. Eine empirische Untersuchung studentischer Texte. Wien/Münster: LIT (= Wissenschaftlich Schreiben. Analyse und Methode 1).

Hinkel, Eli (1997): "Indirectness in L1 and L2 academic writing". Journal of Pragmatics 27/3: 361-386.

Johnson, Ralph H./Blair, J. Anthony (2006): Logical Self-Defense. IDEA, First published, Toronto: McGraw Hill Ryerson, ON, 1997, 1983, 1993. Reprinted, New York: McGraw Hill, 1994.

Kindt, Walther (2008): "Die Rolle sprachlicher Indikatoren für Argumentationsanalysen: Ein Ergebnisbericht aus der Linguistischen Rhetorik“. In: Kreuzbauer, Günther/Gratzl, Norbert (eds.): Rhetorische Wissenschaft: Rede und Argumentation in Theorie und Praxis, Wien: LIT 147-162.

Kluge, Roland (2014). Automatische Argumentanalyse in Webdokumenten zu kontroversen Bildungsthemen. Technische Universität Darmstadt: Master-Thesis.

Liu, Hung Cheng (2000): Zeitausdruck in der Zweitsprache. Eine empirische Untersuchung zum Ausdruck der Temporalität in deutschen Lernersprachen vom Chinesischen. Göttingen: Cuvillier.

Liu, Lu (2005): "Rhetorical education through writing instruction across cultures: A comparative analysis of select online instructional materials on argumentative writing". Journal of Second Language Writing 14/1: 1-18.

Lumer, Christoph (2007): "Überreden ist gut, überzeugen ist besser! Argumentativer Ethos in der Rhetorik”. In: Kreuzbauer, Günther/Gratzl, Norbert/Hiebl, Ewald (eds.): Persuasion und Wissenschaft: Aktuelle Fragestellungen von Rhetorik und Argumentationstheorie. Wien, LIT: 7-33.

Lux, Paul A. (1991): Styles of Anglo and Latin American college student writers. Phoenix: Arizona State University.

Perelman, Chaïm (1979): Logik und Argumentation. Königstein: Athenäum.

Swan, Michael (2005): Practical English Usage. Oxford: Oxford University Press.

Toulmin, Stephen E. (1958): The uses of argument. Cambridge: Cambridge University Press.

Walton, Douglas (2008): “The three bases for the enthymeme: A dialogical theory". Journal of Applied Logic 6/3: 361-379. 
Walton, Douglas (2012): "Using Argumentation Schemes for Argument Extraction: A Bottom-Up Method". International Journal of Cognitive Informatics and Natural Intelligence 6/3: 33-61.

Willard, Charles A. (1996): Liberalism and the Problem of Knowledge: A New Rhetoric for Modern Democracy. Chicago: University of Chicago Press.

Wohlrapp, Harald (2008): Der Begriff des Arguments. Über die Beziehungen zwischen Wissen, Forschen, Glaube, Subjektivität und Vernunft. Würzburg: Königshausen/Neumann.

Woods, John (2000): "How Philosophical is Informal Logic"? Informal Logic 20/2: 139-167.

Zhu, Wei (2001): "Performing argumentative writing in English: Difficulties, processes, and strategies". TESL Canada Journal 19/1: 34-51. 